9. Mak AB, Nixon AM, Moffat J. The mixed lineage leukemia (MLL) fusion-associated gene AF4 promotes CD133 transcription. Cancer Res. 2012;72:1929-34.

10. Wuchter C, Ratei R, Spahn G, Schoch C, Harbott J, Schnittger $\mathrm{S}$, et al. Impact of CD133 (AC133) and CD90 expression analysis for acute leukemia immunophenotyping. Haematologica. 2001;86:154-61.

11. Menendez P, Caballero MD, Prosper F, Del Canizo MC, PerezSimon JA, Mateos MV, et al. The composition of leukapheresis products impacts on the hematopoietic recovery after autologous transplantation independently of the mobilization regimen. Transfusion. 2002;42:1159-72.

12. Yin AH, Miraglia S, Zanjani ED, Almeida-Porada G, Ogawa M, Leary AG, et al. AC133, a novel marker for human hematopoietic stem and progenitor cells. Blood. 1997;90:5002-12.

13. Paulsson K, Lilljebjorn H, Biloglav A, Olsson L, Rissler M, Castor A, et al. The genomic landscape of high hyperdiploid childhood acute lymphoblastic leukemia. Nat Genet. 2015;47:672-6.

14. Quek L, Otto GW, Garnett C, Lhermitte L, Karamitros D, Stoilova $\mathrm{B}$, et al. Genetically distinct leukemic stem cells in human CD34- acute myeloid leukemia are arrested at a hemopoietic precursor-like stage. J Exp Med. 2016;213:1513-35.
15. Agraz-Doblas A, Bueno C, Bashford-Rogers R, Anindita R, Schneider P, Bardini M, et al. Unravelling the cellular origin and clinical prognosis markers of infant B-cell acute lymphoblastic leukemia using genome-wide analysis. Haematologica. 2018; in press.

16. Aldoss I, Bargou RC, Nagorsen D, Friberg GR, Baeuerle PA, Forman SJ. Redirecting $\mathrm{T}$ cells to eradicate B-cell acute lymphoblastic leukemia: bispecific T-cell engagers and chimeric antigen receptors. Leukemia. 2017;31:777-87.

17. Diaconu I, Ballard B, Zhang M, Chen Y, West J, Dotti G, et al. Inducible Caspase-9 selectively modulates the toxicities of CD19Specific chimeric antigen receptor-modified $\mathrm{T}$ cells. Mol Ther. 2017;25:580-92.

18. Pizzitola I, Anjos-Afonso F, Rouault-Pierre K, Lassailly F, Tettamanti S, Spinelli $\mathrm{O}$, et al. Chimeric antigen recptors against CD33/CD123 antigens efficiently target primary acute myeloid cells in vivo. Leukemia. 2014;28:1596-605.

19. Kenderian SS, Ruella M, Shestova O, Klichincky M, Aikawa V, Morrissette JJD, et al. CD33-directed chimeric antigen receptor $\mathrm{T}$ cells exhibit potent preclinical activity against human acute myeloid leukemia. Leukemia. 2015;29:1637-47.

Leukemia (2019) 33:2093-2097

https://doi.org/10.1038/s41375-019-0423-y

Chronic lymphocytic leukemia

\title{
Telomere fusions associate with coding sequence and copy number alterations in CLL
}

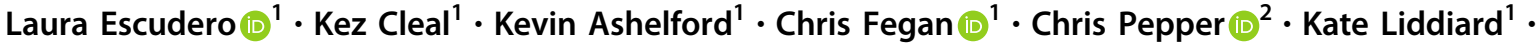 \\ Duncan M. Baird (iD ${ }^{1}$
}

Received: 6 September 2018 / Revised: 19 October 2018 / Accepted: 11 February 2019 / Published online: 22 February 2019

(c) The Author(s) 2019. This article is published with open access

\section{To the Editor:}

Short-dysfunctional telomeres are detected prior to clinical progression in chronic lymphocytic leukaemia (CLL) and result in chromosomal fusions that propagate genome

These authors contributed equally: Kate Liddiard, Duncan M. Baird.

Supplementary information The online version of this article (https:// doi.org/10.1038/s41375-019-0423-y) contains supplementary material, which is available to authorized users.

Duncan M. Baird

bairddm@cf.ac.uk

1 Division of Cancer and Genetics, School of Medicine, Cardiff University, Cardiff, UK

2 Brighton and Sussex Medical School, Sussex University, Brighton, UK instability, driving disease progression. To investigate the impact of telomere dysfunction on the CLL genome, we performed a large-scale molecular characterisation of telomere fusion events in CLL B-cells. A cohort of 276 CLL patient samples was selected for analysis based on short telomere length (TL) profiles, with the majority $(97 \%, n=$ 269) having mean TL within the previously-defined fusogenic range in CLL [1]. Patient samples were screened for the presence of telomere fusions using a single-molecule telomere fusion assay [2] modified to include the 5p telomere (Supplementary Figure 1). Telomere fusions were detected in $72 \%(198 / 276)$ of the samples, which were subsequently arbitrarily stratified by fusion frequency (Supplementary Table 1). Fusions were detected for all telomeres assayed, including the $5 \mathrm{p}$ telomere, for which fusions were present in $23 \%$ (40/177) of patient samples (Supplementary Figure 2, Supplementary Table 2).

High-resolution characterisation of single-molecule amplified telomere fusions from nine CLL patients with 

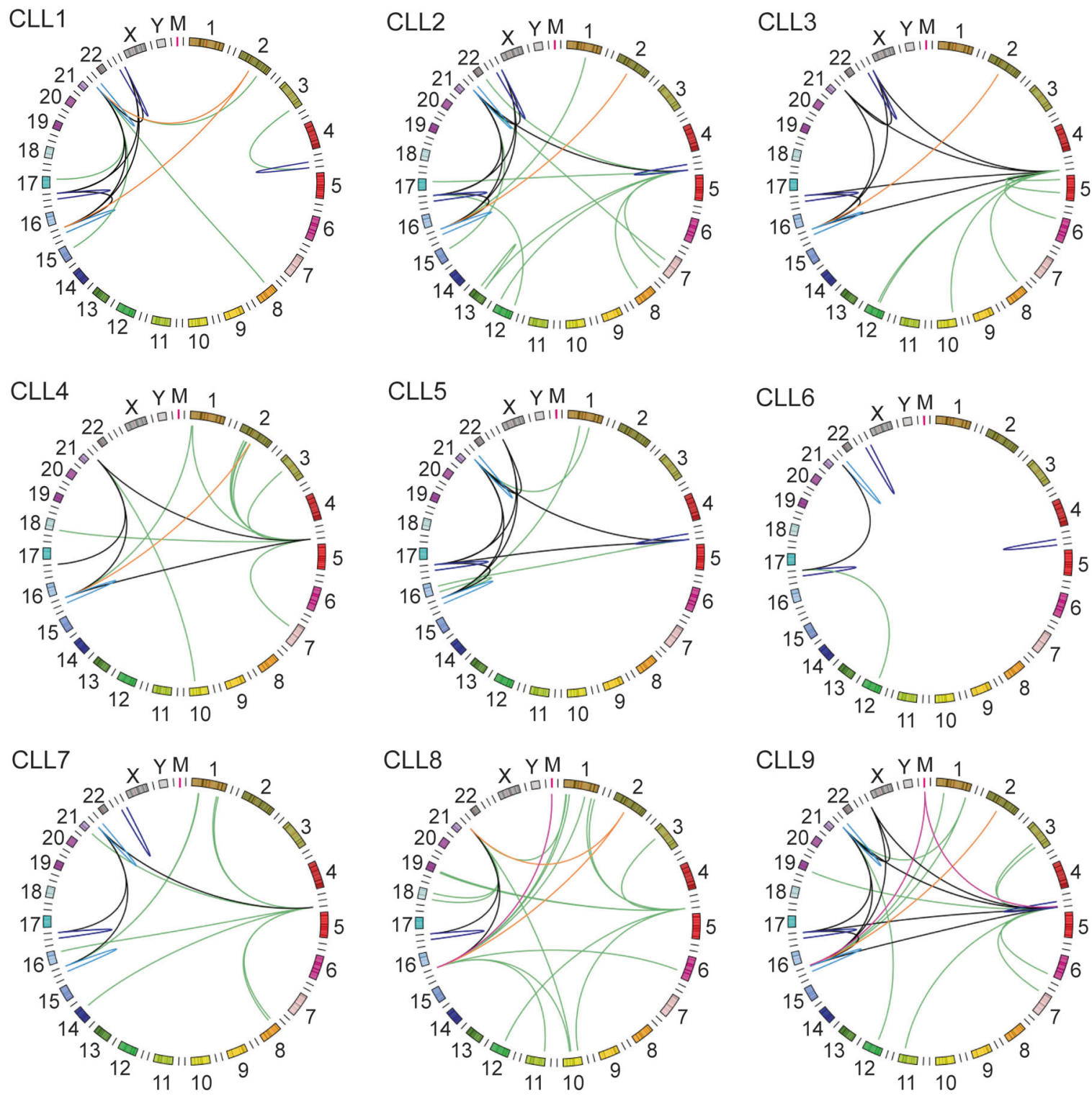

Fig. 1 Signature of telomere fusions for 9 CLL patient samples. Circos plots showing the validated results obtained from the interchromosomal and intra-chromosomal telomere fusion analysis from nine CLL patient samples. Circos plot with each chromosome and its telomeres (1p telomere, Chr1, 1q telomere) around the circle orientated clockwise. Additional notches indicate linkages specifically aligning with subtelomeric sequence references derived from
Stong et al. [12]. Colour code: telomere-telomere inter-chromosomal (black), telomere-telomere intra-chromosomal for 5p, 17p and XpYp (blue), inter-chromosomal or intra-chromosomal for $16 \mathrm{p}$ and $21 \mathrm{q}$ families (light blue), and inter-chromosomal telomere-genomic (green), telomere-2q13 (orange) and telomere-ChrM (pink). Telomere fusion events with unknown sub-telomeric sequence were not included the highest fusion frequency was performed by Illumina HiSeq4000 paired-end sequencing. Following a customised bioinformatics analysis pipeline [3] and manual curation (Supplementary Figure 3), 914 unique telomere fusions were resolved (Supplementary Figure 4), of which 19\% (172/914) involved the $5 p$ telomere (Supplementary Figure 5). Intra(sister-chromatid) or inter-chromosomal telomere fusion events were identified, as well as recombinations involving non-telomeric loci incorporated into telomere fusions (Fig. 1; Supplementary Figure 4, Supplementary Table 3).
These captured loci included; the ancestral telomere at Chr2q13-14 $(n=11)$, mitochondrial DNA $(n=4)$ and other non-telomeric genomic loci $(n=78)$. Complex interchromosomal events involving multiple disparate loci were also detected ( $n=7$ ) (Supplementary Figures 5-9).

Distinct signatures of telomere fusions across the genome could be described for each CLL patient sample (Fig. 1). Two patients (CLL3 and CLL6) displayed simple signatures, defined by the presence solely of intrachromosomal and/or inter-chromosomal telomere-telomere 
fusions. In contrast, the CLL8 sample telomere fusion profile revealed abundant genomic linkages, including with the ancestral telomere at $2 \mathrm{q} 13$ and mitochondrial DNA. Samples CLL1, CLL2, CLL4, CLL5, CLL7 and CLL9 were characterised by complex signatures with a combination of most or all categories of telomere fusion events identified in this study (Fig. 1; Supplementary Table 4).

Telomere dysfunction is associated with increased genomic instability and disease progression in CLL [1, 4], therefore a comprehensive analysis of all patient-derived telomere fusions with non-telomeric genomic loci was undertaken. Locations and junction sequences pertaining to all 93 (10\% total fusions) identified inter-chromosomal fusions were investigated to determine commonality of global or local sequence context as well as providing evidence for the engagement of specific DNA repair processes. These inter-chromosomal genomic fusions were less abundant than pure telomeric inter-chromosomal fusions that represented $38 \%$ of all fusions characterised.

Inter-chromosomal fusions with non-telomeric genomic loci were identified in all nine CLL patient samples. Individual events were validated by manual sequence analysis, revealing 68\% (63/93) had fusion junctions covered by junction-spanning sequence read pairs $(\mathrm{mFJ})$ and $32 \%$ (30/93) had unmapped junctions (uFJ). Each fusion junction location was depicted on the ideogram in Fig. 2a. Notably, the loci disrupted by telomere fusions (summarised in Supplementary Table 5) were not randomly distributed throughout the genome since there was no simple correlation with chromosome length $\left(r^{2}=0.44\right)$ or coding gene density of the respective chromosomes $\left(r^{2}=0.32\right)$ (Fig. $2 b$, Supplementary Figure 10). However, loci with previouslyreported copy number aberrations in CLL [5] were found to be incorporated into telomere fusions, including $2 \mathrm{p} 15$, 2p11.2 (2 events), 2q13 (11 events), 6q22.31, 11q22.2 and 18q21.32 (single events). In addition, a complex telomere fusion was detected involving four distinct loci including 13q14.2 that is frequently deleted in CLL (Supplementary Figure 4B).

Inter-chromosomal telomere fusions occurred within coding DNA more frequently than expected by chance. Over half $(57 \%)$ of $\mathrm{mFJ}$ were within introns and exons of proteincoding genes (Supplementary Table 5), significantly higher (Chi-squared analysis $p=0.0024$ ) than the average $42 \%$ gene content of the human genome (based on the hg19 RefGene). We also observed $15 \%$ and $9 \% \mathrm{mFJ}$ fused with Common Fragile Sites (CFSs) and Alu elements, respectively; however, these were similar to the proportion of CFSs (15\%) and Alu sequences (11\%) identified across the human genome [6].

All 31 protein-coding genes disrupted by telomere fusions with $\mathrm{mFJ}$ were further investigated for potential association with CLL pathogenesis (Supplementary Table 6). An enrichment in genes overexpressed in $\mathrm{CD} 38^{+}$patient
CLL B-cells was revealed using GSEA Gene Set Enrichment Analysis (GSEA, v5.2) Molecular Signatures Database (MSigDB) [7]. This gene set included HTR7, KIF26B and LPHN1 ( $p$-value $1.5 \mathrm{e}^{-6}$; FDR $q$-value $2.7 \mathrm{e}^{-2}$ ) -genes previously found to be upregulated in $\mathrm{CD}^{+} / \mathrm{CD} 19^{+} / \mathrm{CD} 38^{+}$ CLL cells associated with worse patient prognosis, compared with patient-matched $\mathrm{CD}^{-} / \mathrm{CD} 19^{-} / \mathrm{CD} 38^{-}$CLL cells in a panel of six patient samples [8]. Strikingly, 36\% (11/31) of all genes disrupted by a telomere fusion event for which the junction could be validated were classified as expressed or associated with B lymphocytes or CLL B-cells. These genes included CD8A, RORA, TESPA1, DMD, NOX5, NTF3, EVI5 and FTO (Supplementary Table 7) with documented pathological relevance. A significant enrichment in genes possessing binding motifs matching the B-cell-expressed homeobox transcription factor, HNF1 $\alpha$ (TCF1) [9], within their promoters was also identified (DMD, RORA, NTF3 and HTR7; $p$-value $2.51 \mathrm{e}^{-5}$; FDR $q$-value $1.31 \mathrm{e}^{-2}$; Supplementary Table 8). Furthermore, a noteworthy association of fusiondisrupted genes with gene sets over-expressed in other types of cancer including breast and liver was also revealed by these analyses.

We have previously shown that intra-chromosomal telomere fusion is accompanied by extensive resection that results in asymmetric deletion of the participating sister-chromatids [3]. To assess whether this was true for CLL B-cells, the extent of DNA end-processing at each sister-chromatid was examined for intra-chromosomal fusions with $\mathrm{mFJ}$. The distance from the start of the telomere repeat sequences to the fusion junction for each of the chromatids involved in the fusion event was determined and the difference calculated to obtain a measure of asymmetry (Fig. 2c, d; Supplementary Tables 910). The uneven distribution of fusion junctions across the $5 p$ sub-telomere $(n=14)$ is consistent with the location of a $\mathrm{CpG}$ island and suggests that the GC-rich sequence may hamper the detection of $5 p$ fusion events (Supplementary Figure 11). Thus, $5 p$ telomere fusions may be under-represented in the data and may have an even greater impact on CLL disease than presently recognised. In contrast, telomere fusion junctions were effectively captured across the $17 \mathrm{p}(n=30)$ and XpYp $(n=20)$ telomeres (Fig. 2c). Asymmetry of sisterchromatids was observed for $5 \mathrm{p}, 17 \mathrm{p}$ and XpYp with a mean of $1408 \mathrm{bp}, 1240 \mathrm{bp}$ and $695 \mathrm{bp}$, respectively (Fig. 2d). The degree of asymmetry was significantly greater than the theoretical value 0 (one sample $t$-test, $p<0.001$ ). This indicates that fusion occurs between sister-chromatids of different lengths in CLL B-cells, consistent with our observations in other models $[2,3,10]$. No significant differences were found in the extent of asymmetry between the 5p, 17p and XpYp chromosome ends (Kruskal-Wallis, $p=0.1661$ ).

High-resolution analysis of each CLL mFJ was performed to investigate candidate DNA repair mechanisms that may underlie distinct types of telomere fusion events. 

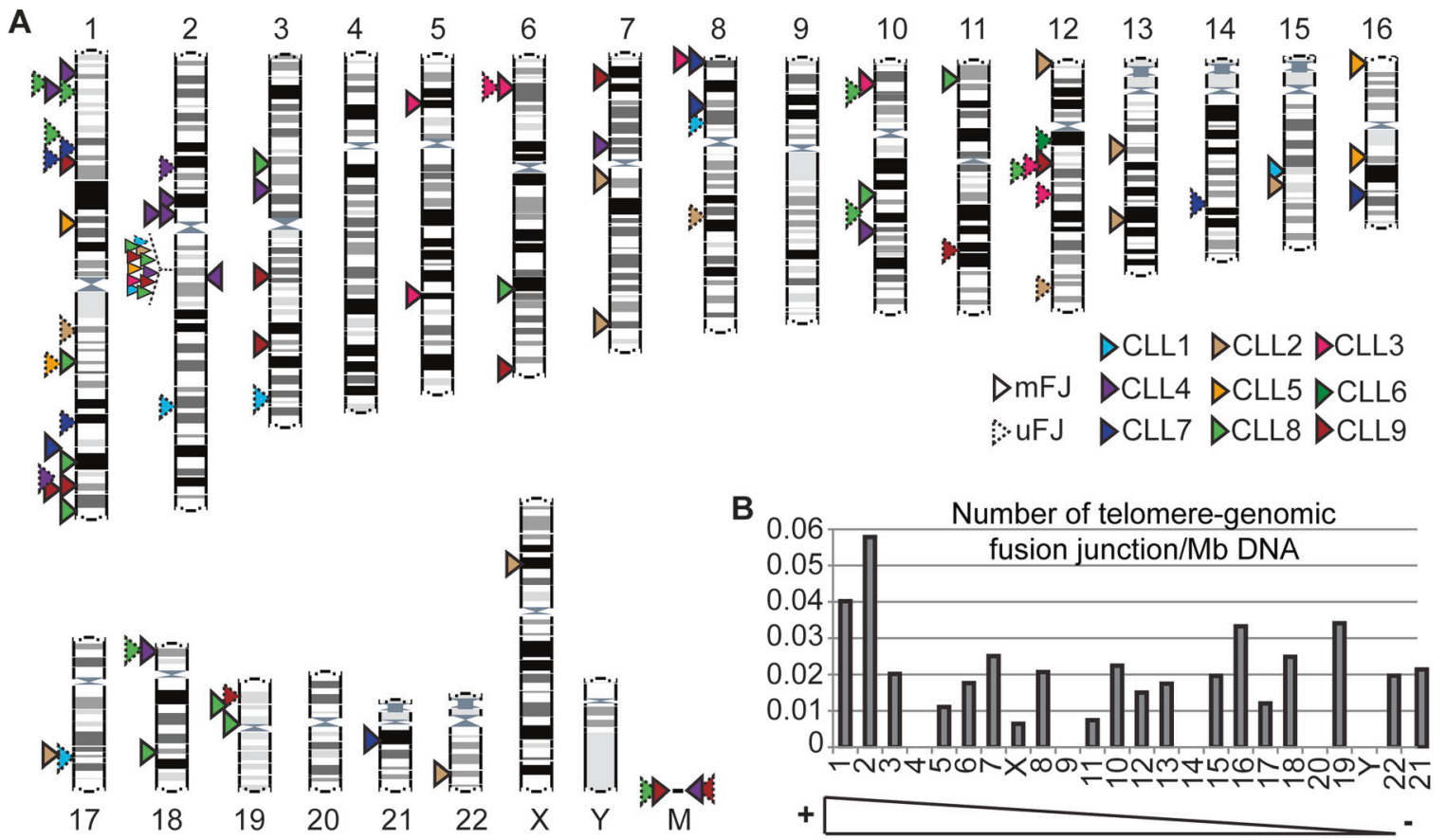
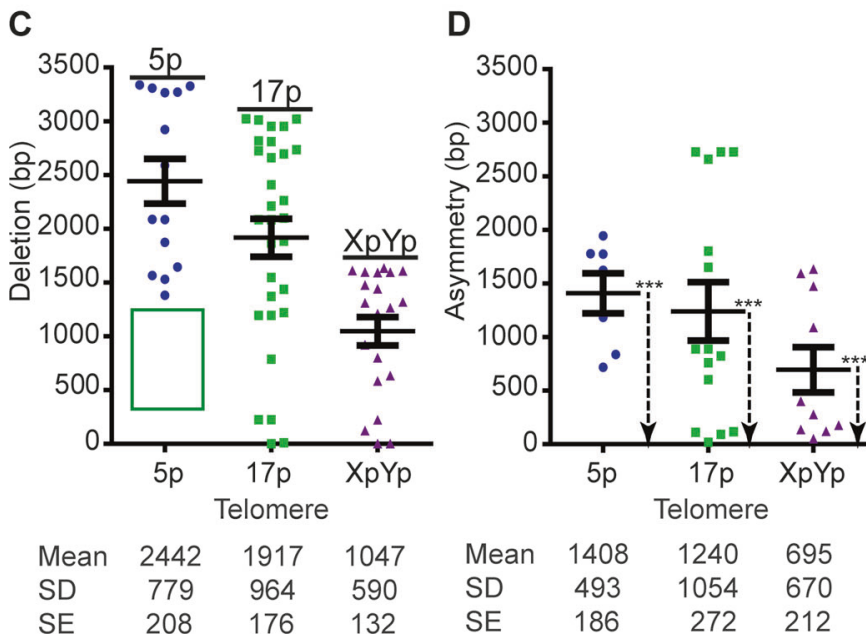

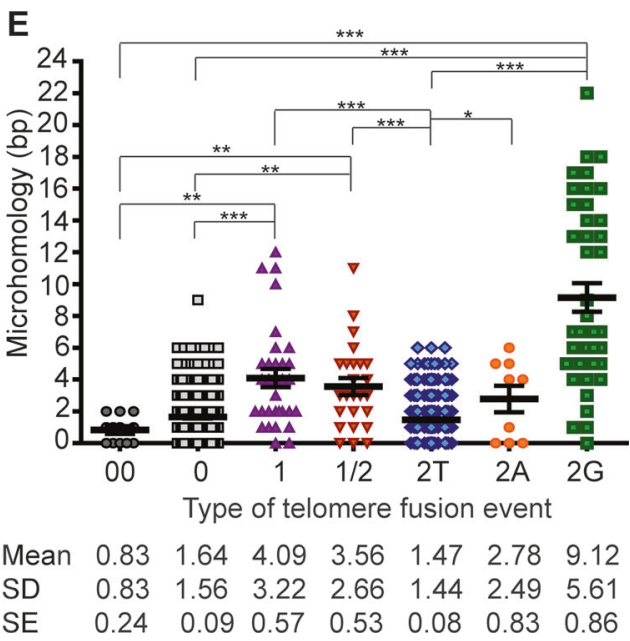

on the $5 \mathrm{p}$ sub-telomere. Location of the fusion primer indicated, determines the limit of the assay from the telomere. d Level of asymmetry was determined by calculating the deletion difference between each chromatid of the same fusion event. e Microhomology (bp) at the fusion junction was compared for the distinct type of events: TTAGGG-CCCTAA (00), Sub-telomere-TTAGGG (0), intrachromosomal (1), intra-chromosoma or inter-chromosomal of $16 \mathrm{p}-16 \mathrm{p}$ and $21 \mathrm{q}-21 \mathrm{q}$ families $(1 / 2)$, inter-chromosomal telomeric fusion events (2T), inter-chromosomal fusions with the ancestral telomere at $2 \mathrm{q} 13$ (2A) and inter-chromosomal fusions with genomic loci (2G). Mean, SD and SE are indicated below

1-21 nucleotides with a mean of 4.5 nucleotides. In contrast, no insertions were identified at fusions with non-telomeric loci. Statistically-significant differences in the extent of microhomology usage at fusion junctions were determined for the different types of telomere fusion events (KruskalWallis $p<0.001$ and Dunn's Multiple Comparison Test) 
(Fig. 2e; Supplementary Table 11). Inter-chromosomal fusions with non-telomeric loci (mean $=9.1 \mathrm{bp} ; n=43$ ), together with intra-chromosomal sister-chromatid events (mean $=4.1 \mathrm{bp} ; n=32$ ), displayed the greatest amounts of junction microhomology. In contrast, very low or an absence of microhomology at the fusion point was observed for inter-chromosomal telomeric fusions (mean $=1.5 \mathrm{bp}$; $n=315$ ), $\quad$ Telomere-Telomere (TTAGGG-CCCTAA; mean $=0.8 \mathrm{bp} ; \quad n=12) \quad$ and Telomere-Sub-telomere (mean $=1.6 \mathrm{bp} ; \quad n=303$ ) subgroups. Long tracts of microhomology of up to $23 \mathrm{bp}$, were observed at interchromosomal fusion junctions with non-telomeric loci (Fig. 2e). When the usage of microhomology was $>10 \mathrm{bp}$, the sequence was enriched for the repeat unit of $(A C)_{n}$ (Supplementary Figure 5); 40\% (6/15) of events that contained at least $(\mathrm{AC})_{5}$ (motif ACACACACAC), consistent with repair utilizing single-stranded annealing [11].

Taken together, our data reveal the impact of shortdysfunctional telomeres on the evolving CLL genome, generating tumour heterogeneity that may affect patient prognosis. We have revealed that dysfunctional telomeres predominantly fuse with protein-coding DNA including genes expressed in CLL B-cells and other tumours. We have also identified complex telomere fusions involving multiple non-telomeric loci across the CLL genome, including those with known copy number aberrations in CLL. Our data implicate diverse DNA repair mechanisms at play in CLL tumour initiation and progression, including C-NHEJ, A-NHEJ and SSA. These repair pathways provide potential therapeutic targets and combinations of therapeutic agents targeting these specific pathway components may effectively sensitise CLL B-cell clones with ongoing telomere dysfunction to improve patient outcomes.

Acknowledgements The authors would like to thank the CLL patients. The study was undertaken with the support of the UK CLL Clinical Trials Sub-Group. This work was supported by Cancer Research Wales, Cardiff University and Cancer Research UK (C17199/A18246). Patient material was obtained from the UK CLL Trials Biobank, University of Liverpool, which is funded by Bloodwise.

Author contributions LE performed the experimental work, analysed the experimental and bioinformatics data and wrote the manuscript; $\mathrm{KC}$ carried out the bioinformatics pipeline; $\mathrm{CF}$ provided clinical samples and edited the manuscript; $\mathrm{CP}$ provided clinical input and edited the manuscript; KL jointly supervised the study and edited the manuscript; DMB jointly supervised the study and edited the manuscript.

\section{Compliance with ethical standards}

Conflict of interest The authors declare that they have no conflict of interest.

Publisher's note: Springer Nature remains neutral with regard to jurisdictional claims in published maps and institutional affiliations.
Open Access This article is licensed under a Creative Commons Attribution 4.0 International License, which permits use, sharing, adaptation, distribution and reproduction in any medium or format, as long as you give appropriate credit to the original author(s) and the source, provide a link to the Creative Commons license, and indicate if changes were made. The images or other third party material in this article are included in the article's Creative Commons license, unless indicated otherwise in a credit line to the material. If material is not included in the article's Creative Commons license and your intended use is not permitted by statutory regulation or exceeds the permitted use, you will need to obtain permission directly from the copyright holder. To view a copy of this license, visit http://creativecommons. org/licenses/by/4.0/.

\section{References}

1. Lin TT, Norris K, Heppel NH, Pratt G, Allan JM, Allsup DJ, et al. Telomere dysfunction accurately predicts clinical outcome in chronic lymphocytic leukaemia, even in patients with early stage disease. Br J Haematol. 2014;167:214-23.

2. Letsolo BT, Rowson J, Baird DM. Fusion of short telomeres in human cells is characterized by extensive deletion and microhomology, and can result in complex rearrangements. Nucleic Acids Res. 2010;38:1841-52.

3. Liddiard K, Ruis B, Takasugi T, Harvey A, Ashelford KE, Hendrickson EA, et al. Sister chromatid telomere fusions, but not NHEJ-mediated inter-chromosomal telomere fusions, occur independently of DNA ligases 3 and 4 . Genome Res. 2016;26:588-600.

4. Lin TT, Letsolo BT, Jones RE, Rowson J, Pratt G, Hewamana S, et al. Telomere dysfunction and fusion during the progression of chronic lymphocytic leukemia: evidence for a telomere crisis. Blood. 2010;116:1899-907.

5. Salaverria I, Martin-Garcia D, Lopez C, Clot G, Garcia-Aragones M, Navarro A, et al. Detection of chromothripsis-like patterns with a custom array platform for chronic lymphocytic leukemia. Genes Chromosomes Cancer. 2015;54:668-80.

6. Fungtammasan A, Walsh E, Chiaromonte F, Eckert KA, Makova $\mathrm{KD}$. A genome-wide analysis of common fragile sites: what features determine chromosomal instability in the human genome? Genome Res. 2012;22:993-1005.

7. Subramanian A, Tamayo P, Mootha VK, Mukherjee S, Ebert BL, Gillette MA, et al. Gene set enrichment analysis: a knowledgebased approach for interpreting genome-wide expression profiles. Proc Natl Acad Sci USA. 2005;102:15545-50.

8. Pepper C, Ward R, Lin TT, Brennan P, Starczynski J, Musson M, et al. Highly purified CD38 + and CD38- sub-clones derived from the same chronic lymphocytic leukemia patient have distinct gene expression signatures despite their monoclonal origin. Leukemia. 2007;21:687-96.

9. von Wnuck Lipinski K, Sattler K, Peters S, Weske S, Keul P, Klump H, et al. Hepatocyte nuclear factor 1A Is a cell-intrinsic transcription factor required for B cell differentiation and development in mice. J Immunol. 2016;196:1655-65.

10. Capper R, Britt-Compton B, Tankimanova M, Rowson J, Letsolo B, Man S, et al. The nature of telomere fusion and a definition of the critical telomere length in human cells. Genes Dev. 2007;21:2495-508.

11. Bhargava R, Onyango DO, Stark JM. Regulation of single-strand annealing and its role in genome maintenance. Trends Genet. 2016;32:566-75.

12. Stong N, Deng Z, Gupta R, Hu S, Paul S, Weiner AK, et al. Subtelomeric CTCF and cohesin binding site organization using improved subtelomere assemblies and a novel annotation pipeline. Genome Res. 2014;24:1039-50. 